\title{
La evolución teórica del marxismo: del materialismo histórico a la crítica de la conciencia fetichista
}

\section{The theoretical evolution of Marxism: from historical materialism to the critique of fetishistic conciousne}

\author{
CÉSAR RuIZ SANJUÁN \\ Universidad Complutense de Madrid
}

\begin{abstract}
RESUMEN. En el presente artículo analizamos en sus aspectos fundamentales la evolución teórica que ha experimentado la interpretación de la obra de Marx desde las primeras corrientes que se derivan de ella hasta las más actuales. Nos ocupamos primero del marxismo tradicional, de su concepción determinista del materialismo histórico y su interpretación historicista de la teoría del valor. Abordamos después las corrientes del marxismo occidental que se oponen a las concepciones reduccionistas y economicistas de la interpretación marxista dominante, para centrarnos finalmente en las interpretaciones más recientes de la teoría social de Marx como crítica del carácter fetichista de las formas de conciencia burguesas y las categorías de la economía política.
\end{abstract}

Palabras clave: Marxismo tradicional, marxismo occidental, teoría del valor, crítica, fetichismo.
ABstract. We analyze in this paper the fundamental aspects of the theoretical evolution that the interpretation of Marx's work has undergone from the first currents of thought derived from it to the most recent ones. First we deal with traditional Marxism, its deterministic conception of historical materialism and its historicist interpretation of the theory of value. Then we consider the currents of Western Marxism opposed to the reductionist and economistic conceptions of prevailing Marxist interpretation, and finally we focus on the most recent interpretations of Marxian social theory as a critique of the fetishistic character of the bourgeois consciousness forms and the categories of political economy.

Key Words: Traditional Marxism, Western Marxism, theory of value, critique, fetishism. 


\section{Introducción}

Uno de los mayores problemas que se presentan al abordar un proceso tan complejo como la recepción del pensamiento de Marx es el hecho de no distinguir con claridad entre las distintas corrientes de interpretación que se han derivado de su obra. Se habla generalmente de "marxismo", en singular, como si la tradición teórica que se deriva de la obra de Marx fuese única y continua. Pero esta tradición ha conocido múltiples transformaciones y variantes a lo largo de su historia, por lo que sería más preciso hablar de "marxismos", en plural, y a partir de aquí definir los caracteres diferenciales de cada uno de ellos. En este trabajo estudiaremos los elementos más relevantes de estas diversas corrientes de interpretación, estableciendo las diferencias esenciales que permiten distinguir entre ellas, lo que pondrá de manifiesto la profunda distancia que las separa en muchos casos.

En una primera aproximación, distinguiremos entre el marxismo tradicional o marxismo ortodoxo, por un lado, y el marxismo occidental o marxismo crítico, por otro lado, realizando una periodización general de ambas corrientes de interpretación y señalando las variantes fundamentales dentro de cada una de ellas. Por marxismo tradicional entendemos la interpretación oficial del pensamiento de Marx, que dominó indiscutidamente desde su muerte hasta la segunda década del siglo $\mathrm{XX}$. A partir de este momento comienzan a surgir las diversas corrientes disidentes de la interpretación oficial, entre las que se encuentran las distintas variantes del denominado marxismo occidental.

El marxismo tradicional o marxismo ortodoxo parte de los escritos de carácter más popular de Marx y de las concepciones tradicionales de la economía política y de la filosofía de la historia, y establece a partir de aquí una concepción economicista y determinista de la sociedad y de la historia. Esta lectura restringida de la teoría de Marx comienza con Engels, se desarrolla en la II Internacional, fundamentalmente bajo la influencia de los escritos de Kautsky, y se consuma con la codificación ideológica del marxismo-leninismo, que ofrece una cosmovisión cerrada y vinculante que se establece como la doctrina oficial del marxismo. El marxismo crítico o marxismo occidental parte de los escritos científicos de la obra de Marx y desarrolla a partir de ellos el análisis y crítica de las relaciones sociales capitalistas y de las formas de conciencia invertida que éstas generan. Buena parte de estas corrientes de interpretación vienen marcadas fundamentalmente por el propósito de "volver a Marx". Para ello es necesario superar las diversas acrecencias ideológicas que se han ido mezclando con su teoría en el desarrollo de la tradición marxista y hacer una lectura de su obra libre de los condicionamientos a los que ha estado sometida en la interpretación dominante. Ello hace preciso, en primer lugar, identificar con claridad esos condicionamientos externos, por lo que el concepto de marxismo tradicional no es simplemente un concepto historiográfico, sino 
que tiene que ser determinado con exactitud para establecer la línea de demarcación que permita definir las distintas posiciones teóricas de las que parte la interpretación ${ }^{1}$.

\section{Surgimiento y desarrollo del marxismo tradicional}

El origen del marxismo tradicional se sitúa en la interpretación de la teoría de Marx realizada por Engels, sobre todo en sus escritos tardíos. Ciertamente, en Engels no aparecen ni el término "marxismo", ni el término "materialismo dialéctico", que es rótulo con el que se ha caracterizado en el marxismo tradicional la concepción filosófica fundamental de Marx. El término "marxismo" aparece por primera vez en 1879 en los escritos de Franz Mehring. Fue también Mehring quien unos años más tarde, en 1893, acuñó el término "materialismo histórico", que es la denominación más ampliamente difundida para referirse a la supuesta filosofía de la historia de Marx que estaría a la base de su análisis y crítica de la sociedad capitalista. El término "materialismo histórico", a diferencia de los términos "materialismo dialéctico" y "marxismo", sí es utilizado por Engels con posterioridad a la introducción de dicho término por Mehring. Fue sobre todo la dificultad de traducción a otras lenguas del término anteriormente utilizado por él de "concepción materialista de la historia" lo que hizo que finalmente acabase aceptando la expresión "materialismo histórico" como término de uso más fluido y más fácil de verter de manera sintética a otras lenguas ${ }^{2}$.

El término "materialismo dialéctico", que es el más ampliamente usado para referirse a la supuesta filosofía general de Marx que estaría a la base de su teoría social, es acuñado por Josef Dietzgen en el año 1887, y su uso se extendió a partir de la sistematización del mismo realizada por Plejanov y Lenin. Este término no aparece en Engels, pero es su determinación de la dialéctica materialista la que

${ }^{1}$ Un caso paradigmático en este sentido es el de M. Postone, que define su posición teórica a partir de una delimitación sistemática respecto al marxismo tradicional. Cf. su obra fundamental Tiempo, trabajo y dominación social: una reinterpretación de la teoría crítica de Marx, Madrid, Marcial Pons, 2006. Igualmente claro es el caso de H.-G. Backhaus, que considera preciso superar las concepciones del marxismo tradicional para acceder a la correcta comprensión de la teoría de Marx, y afirma que "la teoría marxista del valor ha bloqueado necesariamente la comprensión de la teoría marxiana del valor" (Dialektik der Wertform. Untersuchungen zur Marxchen Ökonomiekritik, Freiburg, Ça ira-Verlag, 1997, p. 69).

${ }^{2}$ Véanse a este respecto las consideraciones de Engels en Einleitung zur englischen Ausgabe (1892) der "Entwicklung des Sozialismus von der Utopie zur Wissenschaft", MEW 22, pp. 298 y ss. (Citamos por la edición Karl Marx, Friedrich Engels: Werke (MEW), hrsg. vom Institut für Marxismus-Leninismus beim Zk der SED, Berlin, 1956 ff. En lo sucesivo indicamos solamente las siglas de la edición y el número correspondiente al volumen en que se encuentra la obra). 
establece las bases para la sistematización ulterior de este concepto. Sus escritos tardíos, sobre todo el Anti-Dühring y Ludwig Feuerbach y el fin de la filosofia clásica alemana, se convirtieron en los textos de referencia fundamentales a partir de los que se configuró la interpretación oficial del marxismo ${ }^{3}$.

Engels entiende la dialéctica como "la ciencia de las leyes generales del movimiento y la evolución de la naturaleza, la sociedad humana y el pensamiento"4. Estas leyes se reducen básicamente a la negación de la negación, el paso de la cantidad a la cualidad y la interpenetración de los opuestos ${ }^{5}$. Las leyes dialécticas, por tanto, rigen todos los procesos reales, tanto de la naturaleza como de la historia, y este respecto de la dialéctica es denominado por Engels "dialéctica objetiva". Frente a ella, se encuentra lo que Engels llama "dialéctica subjetiva", que entiende como el reflejo en la mente humana de las leyes dialécticas que operan en el ámbito de la naturaleza y de la historia. La dialéctica subjetiva queda subordinada, por tanto, a la dialéctica objetiva, y esta subordinación es la base de una epistemología realista y puramente contemplativa, según la cual la mente humana se limitaría a reproducir de manera pasiva la realidad.

Esta forma de plantear la cuestión de la dialéctica tiene implicaciones fundamentales sobre aquello que se entiende por materialismo. Engels, y tras él todo el marxismo ortodoxo, entiende por materialismo la primacía de lo material sobre lo espiritual, llevando a cabo con ello la supuesta inversión de Hegel a la que se refiere Marx en algunas ocasiones. Pero este planteamiento ontológico no es el que opera en la comprensión de Marx del materialismo. Como pondrán de manifiesto algunos de los principales pensadores del marxismo occidental, lo que Marx entiende por materialismo es la dependencia de la conciencia respecto de las condiciones sociales de existencia, lo que no lleva consigo en modo alguno una epistemología realista según la cual el sujeto se limita a reflejar pasivamente la realidad exterior a él, sino que el sujeto lleva a cabo una refiguración teórica de la realidad a partir de categorías que no tienen ningún correlato empírico inmediato ${ }^{6}$. La concepción materialista de Marx le lleva a desvelar el condicionamiento de la conciencia respecto de las condiciones materiales de vida, para llevar a cabo a partir de aquí una crítica tanto de la realidad social, cuyas estructuras fundamentales

\footnotetext{
${ }^{3}$ En este sentido afirma Ingo Elbe que el "marxismo es en muchos aspectos obra de Engels, por lo que es propiamente un engelsismo" (Marx im Westen, Berlin, Akademie Verlag, 2008, p. 14).

${ }^{4}$ La subversión de la ciencia por el señor Eugen Dühring (Anti-Dühring), Barcelona, Crítica, 1977, p. 144.

${ }^{5}$ Cf. Dialéctica de la naturaleza, Madrid, Akal, 1978, pp. 58 y ss.

${ }^{6}$ Esta concepción se presenta con especial claridad en el texto metodológico fundamental de Marx, la Introducción a la crítica de la economía política de 1857. Cf. Elementos fundamentales para la crítica de la economía política (Grundrisse) 1857-1858 (volumen 1), México, Siglo XXI, 2001, pp. 2-33.
} 
permanecen veladas por la ideologización de la conciencia sometida a ellas, como una crítica de esa conciencia invertida con el objeto de liberarla de esos condicionamientos a los que se halla sometida. Lo que se deriva de aquí, por tanto, es un análisis y una crítica de sociedad y de las formas de conciencia social, pero no una cosmovisión que pretenda explicar todos los ámbitos de lo real, tal y como es el caso del marxismo tradicional, que acaba derivando necesariamente en mera ideología que pretende ofrecer una concepción global del mundo en su totalidad.

El denominado "socialismo científico" de la II Internacional que se desarrolla a partir de los planteamientos de Engels se convierte así en un sistema ontológico que pretende explicar todos los ámbitos de lo real, tanto la naturaleza como la historia. La interpretación de Engels de la dialéctica en clave materialista consiste básicamente en aplicar las supuestas leyes dialécticas universales tanto a los procesos naturales como a la evolución histórica, estableciendo una doctrina universal del ser y cortando a la misma escala determinista tanto los fenómenos naturales como los históricos. Ello es consecuencia, como luego hará patente la crítica de Lukács, de la ausencia de mediación subjetiva en la concepción de la dialéctica de Engels, que se convierte en un objetivismo del que queda evacuada cualquier posible relación sujeto-objeto. La interpretación engelsiana de la dialéctica va dirigida contra la concepción estática de lo real, tratando de captar la dimensión de flujo y transformación de los procesos reales. Frente a esta dialéctica objetiva, la dialéctica subjetiva no sería más que una copia pasiva en la mente humana de este proceso de cambio de la realidad objetiva. Ello acaba derivando necesariamente en un realismo ingenuo, que será sistematizado por Lenin en la "teoría del reflejo"7, según la cual el pensamiento se limita a la reproducción pasiva de la realidad exterior. La pretensión fundamental de Lenin es oponer al carácter estático del materialismo metafísico, el carácter dinámico del materialismo dialéctico, lo que se hace al precio de una concepción determinista a nivel ontológico y de un craso realismo a nivel epistemológico. El retén que todavía existía en el pensamiento de Engels, cuyo talante crítico le impide llegar a estos excesos dogmáticos, queda ya definitivamente eliminado en la interpretación de Lenin ${ }^{8}$.

Esta sistematización de Lenin se establece como la concepción básica del marxismo ortodoxo, según la cual el conocimiento es una simple reproducción especular de lo real, la naturaleza se encuentra dominada por un mecanicismo de fuerzas naturales en oposición y la historia regida por una dialéctica de fuerzas pro-

\footnotetext{
${ }^{7}$ Para la exposición más completa de esta teoría por parte de Lenin, véase Materialismo y empiriocriticismo, Madrid, Ayuso, 1974.

${ }^{8}$ Engels había insistido en la escasa capacidad explicativa de tales leyes generales y en la necesidad del estudio empírico de los fenómenos particulares para alcanzar una explicación científica de los mismos. Cf. Anti-Dühring, cit., pp. 144 y ss.
} 
ductivas y relaciones de producción. Todo este proceso de regresión teórica se consuma en el denominado Diamat, el "materialismo dialéctico" elevado por Stalin a cosmovisión vinculante del movimiento obrero internacional, en tanto que doctrina oficial del Partido Comunista soviético. De la aplicación a la historia de este materialismo dialéctico resulta el materialismo histórico, rótulo bajo el que se engloba la concepción de la sociedad y de la historia del marxismo tradicional. Con ello la teoría crítica de la sociedad de Marx se acaba convirtiendo en una doctrina mecanicista y determinista de la evolución histórica de las sociedades. Bajo la presunta cientificidad de esta doctrina no se oculta otra cosa que una metafísica de la historia que anula por completo la dimensión crítica de la teoría social de Marx.

En la interpretación del "materialismo histórico", esta concepción de la historia sería la que estaría a la base de la teoría de la sociedad de Marx, por lo que esta teoría no sería en realidad más que un elemento parcial de una concepción más amplia de la evolución histórica de las sociedades que Marx ciertamente nunca llegó a elaborar de manera completa, pero cuyas líneas de fuerza fundamentales estarían determinando el sentido de su análisis de la sociedad capitalista. Los textos de Marx en los que la tradición marxista apoya esta concepción se limitan básicamente a algunos pasajes del comienzo de La ideología alemana ${ }^{9}$ y, sobre todo, al famoso Prólogo a la Contribución ${ }^{10}$, cuyas escasas cinco páginas de imprenta son citadas hasta la saciedad en el marxismo ortodoxo, y ello precisamente porque no le es posible encontrar en la obra de Marx otro texto en el que pueda apoyar su interpretación. De este escuálido apoyo textual deriva el marxismo tradicional una teoría general del curso histórico según la cual las distintas formas de sociedad se suceden unas a otras regidas por una férrea legalidad económica, siendo la estructura económica la única instancia determinante de esa evolución y careciendo de papel alguno en este proceso las demás esferas de la vida social, las cuales carecen de toda sustantividad y están determinadas completamente por la base económica del respectivo modo de producción. En cualquier caso, una lectura detenida de estas páginas pone de manifiesto que es perfectamente posible interpretarlas de una manera sustancialmente distinta a ésta, e incluso Engels se opuso a semejantes interpretaciones estrictamente economicistas de la concepción materialista de la historia, de las que ciertamente su propia interpretación contenida en los textos tardíos constituye la base conceptual, pero a las que se niega a seguir hasta este extremo de degradación teórica. Para ello recurre a fórmulas ciertamente problemáticas como la de "determinación en última instancia" "11, pero que en cual-

\footnotetext{
${ }^{9}$ Cf. La ideología alemana, Barcelona, Grijalbo, 1974, pp. 15-90.

${ }^{10}$ Cf. Contribución a la crítica de la economía política, México, Siglo XXI, 1998, pp. 3-7.

${ }^{11}$ Sobre esta cuestión insistió Engels con particular claridad en la carta a J. Bloch del 21/09/1890, MEW 37, pp. 463 y ss.
} 
quier caso son sintomáticas de la dimensión crítica que aún alberga el pensamiento del Engels tardío, frente a la vocación definitivamente ideológica que marcará el curso del marxismo ortodoxo.

\section{La historización de la teoría del valor}

Pero la interpretación de Engels de la obra de Marx no sólo determina la concepción del materialismo histórico, sino también la comprensión de la crítica marxiana de la economía política, fundamentalmente de la estructura metodológica de $\mathrm{El} \mathrm{Ca}$ pital y del sentido de la teoría del valor ${ }^{12}$. A este respecto, Engels historiza la teoría del valor de Marx, al determinar que el método aplicado por éste es un método "histórico-lógico". Según esta concepción del método que opera en $E l \mathrm{Ca}$ pital, se entiende que Marx lleva a cabo una exposición lógica del sistema capitalista, esto es, una exposición en la que las diversas categorías se siguen unas a otras según un orden lógico, pero que esta exposición lógica se corresponde con el proceso histórico de surgimiento y desarrollo del sistema capitalista, de modo que el orden lógico no sería más que el reflejo abstracto del proceso histórico. Esta concepción del método aplicado por Marx en El Capital dominará de manera indiscutida en el marxismo tradicional, obturando por principio la comprensión adecuada del método de Marx. Esta comprensión del método también ha tenido una fuerte influencia en muchos autores destacados del marxismo occidental ${ }^{13}$.

A partir de esta concepción del método, Engels entiende la teoría del valor como una exposición histórica de lo que él denomina "producción mercantil simple". Se trataría de un tipo de sociedad histórica anterior a la sociedad capitalista, a partir de la cual se habría desarrollado ésta. La interpretación historicista de Engels implica que en la teoría del valor, Marx estaría analizando economías precapitalistas en las que tiene lugar el intercambio de mercancías regido por los valores de las mismas, de modo que en ellas se cumpliría plenamente la ley del valor. Aquí podrían los productores medir directamente el tiempo de trabajo invertido en la producción de las mercancías que intercambian. El dinero no desempeña ningún papel en esta interpretación de Engels. De hecho, la ley del valor tendría plena va-

${ }^{12}$ Los textos de Engels que determinaron la comprensión de la crítica de la economía política de Marx fueron básicamente su Recensión a la Contribución y su Apéndice al libro III de El Capital. Cf. Engels, F., "La Contribución a la crítica de la economía politica de Karl Marx”, en Marx, K., Contribución a la crítica de la economía política, cit., pp. 333-343, y “Apéndice y notas complementarias al tomo III de El Capital", en Marx, K., El Capital. Crítica de la economía política. Libro III, México, Siglo XXI, 1996, pp. 1125-1150.

${ }^{13}$ Véase, por ejemplo, Zeleny, J., La estructura lógica de "El Capital” de Marx, Barcelona, Grijalbo, 1974; o Mandel, E., Tratado de economía marxista, México, Era, 1976. 
lidez, según él, en épocas premonetarias. Esta interpretación de Engels supone una historización de las categorías económicas con las que opera Marx en El Capital, y ello no sólo en el sentido de que resultan aplicables a otras sociedades anteriores a la sociedad capitalista, sino incluso en el sentido teóricamente más fuerte de que las categorías que se presentan en la teoría del valor (precisamente las categorías que constituyen el núcleo mismo del instrumentario teórico de El Capital) tienen su plena validez en sociedades preburguesas. Estas sociedades denominadas por Engels de "producción mercantil simple" habrían durado hasta finales de la Edad Media, y en ellas los individuos podían determinar el valor de los productos que intercambiaban a través de un cómputo directo del tiempo de trabajo invertido en su producción. Sería precisamente el desarrollo del capital lo que no haría posible ya dicho cómputo directo, lo que introduciría modificaciones en la ley del valor, que dejaría de tener plena validez en la sociedad capitalista.

A este respecto hay que señalar, por un lado, que Marx afirma explícitamente en El Capital que la ley del valor "se desarrolla libremente, por primera vez, sobre el fundamento de la producción capitalista"14; y por otro lado, que Marx no ha utilizado en ningún lugar de su obra la expresión "producción mercantil simple" (einfache Warenproduktion). El término que utiliza Marx repetidamente para referirse al nivel de exposición que desarrolla en la primera sección de El Capital, en la que aparecen las categorías fundamentales de la teoría del valor, es el de "circulación mercantil simple" (einfache Warenzirkulation). El desplazamiento conceptual que opera Engels al sustituir el concepto de "circulación" por el de "producción" supone precisamente establecer una separación entre el modo de producción que se estaría tematizando en la primera sección de El Capital y el modo de producción que sería objeto de análisis a partir de la segunda sección de la obra, en que se presenta por primera vez la categoría de capital. Pero para Marx se trata en todo momento del análisis del modo de producción capitalista, y a lo que se refiere con el término "circulación mercantil simple" es a una esfera abstracta del modo de producción capitalista, esto es, a la esfera de la circulación o intercambio de mercancías de la sociedad capitalista abstrayendo de la esfera de la producción, por lo que se exponen la mercancía, el valor y el dinero sin hacer referencia explícita al capital ${ }^{15}$. Y este modo de exposición es obligado por las condiciones mismas que impone el objeto

${ }^{14}$ El Capital. Crítica de la economía politica. Libro I, México, Siglo XXI, 1995, p. 652 (Cuando en lo sucesivo citemos El Capital, sin más especificaciones, nos referimos siempre al libro I).

${ }^{15} \mathrm{Si}$ bien Marx no tematiza esta cuestión en El Capital, sí lo hace en distintos lugares de los manuscritos, fundamentalmente en los Grundrisse y en el Urtext. Véase, por ejemplo, Elementos fundamentales para la crítica de la economía política (volumen 1), cit., pp. 194 y ss., o "Fragmento de la versión primitiva de la Contribución a la crítica de la economía política", en Elementos fundamentales para la crítica de la economía política (volumen 3), México, Siglo XXI, 2002, pp. 183 y ss. 
de investigación, la sociedad capitalista, en la que el intercambio de mercancías y dinero aparece como un ámbito separado e independiente de la producción. En esta esfera de la circulación se presentan los individuos como libres e iguales, en tanto que su actividad consiste en intercambiar con entera libertad mercancías que tienen el mismo valor, por lo que no puede existir desigualdad ni explotación. Puesto que esta esfera de la circulación de mercancías genera la apariencia ideológicamente funcional que está a la base de la economía política y de la conciencia burguesa, la teoría de Marx parte de ella como primer nivel de la exposición, para realizar una crítica inmanente de dichas formas de conciencia y de la sistematización teórica de las mismas que lleva a cabo la teoría económica burguesa.

Así pues, frente a la construcción lógica con un propósito crítico que desarro1la Marx, Engels plantea una construcción histórico-lógica con un propósito básicamente descriptivo. Esta forma de interpretación historicista y empirista de $E l$ Capital domina de manera casi indiscutida hasta los años 60 del siglo XX. Lo que tiene lugar aquí es una transformación de la crítica de la economía política de Marx en una economía política socialista ${ }^{16}$. Esta concepción se le adscribió de manera indiscriminada a Marx, y en general los críticos de su teoría dirigieron sus objeciones contra ella bajo el supuesto de que con ello criticaban al mismo Marx. Algunos autores realizaron un análisis más diferenciado, pero en última instancia partieron todos ellos de la identidad de planteamientos de Engels y Marx, lo que hizo que la teoría de Marx fuera interpretada bajo el prisma de la concepción de Engels. Si bien hubo ciertos teóricos que de manera excepcional se opusieron a esta interpretación, no ejercieron influencia alguna frente a la concepción dominante ${ }^{17}$.

\section{El marxismo occidental y la crítica del marxismo tradicional}

El marxismo occidental tiene su origen en los acontecimientos que sucedieron a la crisis del movimiento obrero internacional en el contexto de la I Guerra Mundial, con el establecimiento de un ala socialdemócrata y un ala comunista, el fin de la II Internacional y el triunfo de la Revolución bolchevique en Rusia ${ }^{18}$. Los escritos de Lukács y de Korsch, que aparecieron simultáneamente en el año 1923,

${ }^{16}$ Esta forma de interpretación ha sido calificada por Robert Kurz como "socialismo adjetivo", por la pérdida del núcleo verdaderamente sustantivo de la crítica marxiana que tiene lugar en ella.

${ }^{17}$ Es el caso del autor ruso Isaac Rubin, véase Ensayos sobre la teoría marxista del valor, Buenos Aires, Pasado y Presente, 1974. En esta obra, publicada originalmente en 1928, se anticipan muchas cuestiones que serán objeto de análisis del marxismo crítico cuatro décadas después.

${ }^{18}$ El término surge en el contexto de la polémica de los leninistas contra Lukács, pero su uso es sistematizado por P. Anderson en la obra Consideraciones sobre el marxismo occidental, México, Siglo XXI, 1991. 
marcaron el inicio de esta nueva corriente del marxismo disidente de la interpretación oficial ${ }^{19}$. Lukács fue el primer autor marxista en criticar la identidad de los planteamientos de Marx y Engels, realizando una sólida crítica a la concepción objetivista de la dialéctica de Engels y señalando las diferencias fundamentales que la separaban de la concepción de Marx. Lukács se opone fundamentalmente a la existencia de leyes objetivas de la evolución histórica y al realismo ingenuo del marxismo ortodoxo. Sus planteamientos están dirigidos a la crítica de la cosificación de la conciencia que tiene lugar en las condiciones capitalistas, y a partir de ellos desarrolla una teoría de la praxis social de orientación revolucionaria. La categoría teórica central de Lukács, que tanta influencia tendrá en el curso del marxismo occidental, es la de enajenación (Entfremdung), que se deriva de la cosificación a la que da lugar la producción mercantil. La burguesía permanece necesariamente atrapada en la enajenación, y es únicamente el proletariado el que puede llevar a cabo la transformación de esta condición estructural de la sociedad capitalista, adquiriendo conciencia de su estado enajenado y transformándose en el sujeto consciente del proceso histórico. En la estela abierta por la interpretación hegelianizante de Lukács se desarrollarán los planteamientos marxistas de autores como Merleau-Ponty, Kosík, Lefèbvre, Bloch o los teóricos de la Escuela de Frankfurt, cuyas interpretaciones tienen como denominador común la proyección de las categorías de la filosofía hegeliana en la teoría social de Marx.

Con la imposición del marxismo-leninismo como doctrina oficial de la III Internacional, tiene lugar ya desde su mismo origen el aislamiento de los teóricos del marxismo occidental, que será una constante que marcará a todos los sucesivos autores que se inscriben dentro de esta corriente, cuya influencia en el ámbito intelectual es inversamente proporcional a su influencia en el ámbito de la política. Los aspectos fundamentales que caracterizan a estas corrientes del marxismo crítico son la preocupación por cuestiones metodológicas relativas a la crítica de la economía política de Marx, la investigación de la relación entre Marx y Hegel, en cuyo pensamiento buscarán muchos de ellos los elementos teóricos para la comprensión de la teoría marxiana, y el interés por los textos del joven Marx, cuya dimensión humanista opondrán a la orientación economicista del marxismo ortodoxo ${ }^{20}$. Esta confrontación resultará enormemente fructífera a nivel teórico, consiguiendo liberar la interpretación de Marx de buena parte de los prejuicios y

\footnotetext{
${ }^{19}$ Cf. Lukács, G., Historia y conciencia de clase, Barcelona, Grijalbo, 1976; Korsch, K., Marxismo y filosofía, Barcelona, Ariel, 1978.

${ }^{20}$ El texto de referencia fundamental en este sentido serán los "Manuscritos económico-filosóficos", escritos por Marx en París en 1844, pero que permanecieron inéditos durante su vida y fueron publicados por primera vez en 1932. Cf. Manuscritos: economía y filosofía, Madrid, Alianza, 1997.
} 
condicionamientos a los que estaba sometida bajo el predominio del marxismoleninismo. Pero el nivel de generalidad al que se movió la polémica, la orientación fundamentalmente hegeliana de muchos de sus mayores representantes, así como la insistencia en recuperar los aspectos humanistas del pensamiento de Marx, establecieron límites insoslayables a la comprensión del núcleo teórico y de la estructura metodológica de la crítica marxiana de la economía política. De hecho, ninguno de los grandes autores del marxismo occidental establece una confrontación de fondo con la interpretación de la teoría del valor dominante en el marxismo tradicional.

Esta confrontación no tendrá lugar hasta mediados de la década de 1960. En estos momentos surgen una serie de interpretaciones dentro del marxismo occidental que pretenden superar la comprensión limitada de la estructura metodológica de la crítica marxiana de la economía política y de su núcleo fundamental, constituido por la teoría del valor. Se presenta aquí una crítica de la interpretación historicista y empirista de la teoría del valor de Marx que había dominado en el marxismo ortodoxo. El principal punto de ruptura en este sentido lo constituye la impugnación de la comprensión sustancialista del valor que sostenía el marxismo tradicional, que viene a sumarse al rechazo de las interpretaciones de la crítica de la economía política centradas en el movimiento obrero y en la revolución prevalecientes en la tradición marxista.

Los primeros planteamientos de esta nueva interpretación tienen lugar en el coloquio internacional "100 años de El Capital", que se celebró en 1967 bajo la égida de Alfred Schmidt, y en el que participaron otros importantes autores del marxismo occidental como Mandel, Poulantzas o Rosdolsky ${ }^{21}$. La nueva interpretación que surge aquí se centra en la cuestión del método de la crítica de la economía política y en la dimensión crítica de la teoría social de Marx. Se aborda por primera vez con claridad la importancia central de las determinaciones económicas formales en la teoría del valor, así como los elementos fundamentales del modo de exposición de Marx, analizando la función que tiene en él la dialéctica, cuya dimensión metodológica en la crítica de la economía política se toma en consideración por primera vez, frente a las interpretaciones generalistas de la dialéctica que habían dominado tanto en el marxismo tradicional como en el marxismo occidental. Otro elemento novedoso que se presenta en este momento, y que constituirá una constante en todos los autores que se adscriben a esta nueva corriente de interpretación, es la importancia que se le concede a los Grundrisse y a otros manuscritos preparatorios de El Capital para comprender la estructura teórica de la

${ }^{21}$ Schmidt, A.; Euchner, W. (eds.), Kritik der politischen Ökonomie heute. 100 Jahre "Kapital”, Frankfurt a. M., Europäische Verlagsanstalt, 1968. 
crítica de la economía política ${ }^{22}$. Se presenta aquí también la influencia de la interpretación estructuralista desarrollada por Althusser, que puso de manifiesto cuestiones que tendrían una importancia fundamental en todos los debates posteriores, como la diferencia irreductible que se presentaba en la obra de madurez de Marx entre el orden del conocimiento y orden de lo real, o el profundo sentido teórico de la crítica de Marx a la economía política, que supone no sólo la crítica de las teorías económicas burguesas o de su objeto de investigación, sino la crítica de los fundamentos teóricos mismos de la economía política y de su carácter de ciencia, señalando asimismo a la existencia de una reflexión insuficiente por parte de Marx respecto al verdadero alcance de su empresa teórica ${ }^{23}$.

En este contexto teórico, surgen toda una serie de autores que impugnan no sólo la concepción de Engels del materialismo histórico, sino también su comprensión de la crítica marxiana de la economía política. En un primer momento se opone la teoría marxiana del valor a la teoría marxista del valor originada en la interpretación de Engels. Después se cuestiona la autocomprensión de Marx de su propio desarrollo teórico, bajo la influencia de la interpretación de Althusser. Finalmente se distingue entre los estratos exotéricos y los esotéricos en la teoría de Marx, iniciándose también el debate sobre la popularización de su propia teoría por parte de Marx y sobre la progresiva ocultación del método que tiene lugar en las sucesivas versiones de la crítica de la economía política ${ }^{24}$.

En la interpretación que desarrollan estos autores, la teoría marxiana se entiende como análisis y crítica del contexto social cosificado y de las formas de conciencia fetichista que genera la mediación material de las relaciones sociales en el sistema capitalista. El análisis y desarrollo de las formas económicas se comprende como el núcleo teórico de la crítica marxiana de la economía política. Este desarrollo no tiene carácter histórico, sino que lleva a cabo la descodificación teórica de la estructura de la sociedad capitalista. Desvela el carácter aparentemente ob-

${ }^{22}$ La primera obra importante en la que los Grundrisse tienen un papel relevante en la interpretación es Der Begriff der Natur in der Lehre von Marx, publicada en 1962 por A. Schmidt como reelaboración de su tesis doctoral, dirigida por Adorno y Horkheimer. Cf. El concepto de naturaleza en Marx, México, Siglo XXI, 1983. Pero es la obra de R. Rosdolsky Zur Entstehungsgeschichte des Marxschen "Kapital": Der Rohentwurf des "Kapital" 1857-1858, publicada en 1968, la que pone definitivamente los Grundrisse en el centro de la investigación de la obra marxiana. Cf. Génesis y estructura de "El Capital" de Marx. Estudios sobre los "Grundrisse", México, Siglo XXI, 1985.

${ }^{23}$ Las dos obras fundamentales de Althusser, Pour Marx y Lire le Capital, habían sido publicadas en 1965. Cf. La revolución teórica de Marx, México, Siglo XXI, 1987 y Para leer "El Capital”, México, Siglo XXI, 1978.

${ }^{24}$ Una visión de conjunto de esta evolución puede verse en Elbe, I., Marx im Westen, cit., pp. 12-29. 
jetivo y autónomo de las formas económicas como formas de praxis social específicamente históricas, y por ello en principio transformables.

\section{El marxismo como crítica de la conciencia fetichista}

Buena parte de los autores que desarrollaron su interpretación en la línea anteriormente señalada se agruparon posteriormente bajo el rótulo de "nueva lectura de Marx"25. Estos intérpretes abordan en profundidad la teoría del valor de Marx desde un punto de vista cualitativo, considerando que Marx no expone simplemente una teoría dirigida a cuantificar el valor en términos de trabajo, sino que más bien trata de analizar las condiciones y la lógica de un contexto social en el que los individuos se ven obligados estructuralmente a intercambiar los productos del trabajo como mercancías para que su trabajo valga como social, y se constituya así en la sustancia del valor en tanto que trabajo abstractamente humano. En este proceso de mediación material de las relaciones sociales se generan ineludiblemente toda una serie de representaciones invertidas y de mistificaciones a las que queda sometida la conciencia de todos los agentes, independientemente de su posición social. No se considera ya la crítica marxiana de la economía política como una teoría realizada desde el punto de vista de la clase trabajadora, tal y como había sido usual en el marxismo tradicional y en buena parte del marxismo occidental, sino como un análisis y crítica de la conciencia fetichista a la que sucumben todos los individuos integrados en las relaciones sociales capitalistas, independientemente de la clase a la que pertenezcan.

Estos autores comienzan a comprender la importancia que para esta dimensión crítica de la teoría marxiana tiene el análisis de la forma de valor y el desarrollo lógico que opera a este nivel de la exposición de Marx ${ }^{26}$. Pero en sus investigaciones metodológicas constatan también lo problemática que resulta esta dimensión de la teoría de Marx: en primer lugar, por el carácter fragmentario de la misma, pues Marx no llegó a concluir su crítica de la economía política en los térmi-

\footnotetext{
${ }^{25}$ Esta denominación es introducida por Backhaus en 1997, y con ella engloba retrospectivamente a toda una serie de autores que desde finales de la década de los 60 hasta la actualidad estaban planteando su interpretación desde coordenadas teóricas comunes. Véase Dialektik der Wertform, cit., pp. 10 y ss.

${ }^{26}$ Los títulos de los primeros trabajos de Backhaus y Reichelt, que son los autores con los que esta corriente de interpretación comienza a cobrar una fisionomía definida, constituyen a este respecto toda una declaración de principios: "Sobre la dialéctica de la forma de valor" y "Sobre la estructura lógica del concepto de capital en Karl Marx", respectivamente. Cf. Backhaus, H.G., "Zur Dialektik der Wertform”, en Schmidt, A. (ed.), Beiträge zur marxistischen Erkenntnistheorie, 1969 y Reichelt, H., Zur logischen Struktur des Kapitalbegriffs bei Karl Marx, Freiburg, Ça-Ira Verlag, 1970.
} 
nos en que la tenía proyectada; en segundo lugar, por las distintas versiones que presenta del núcleo constitutivo de su crítica de la economía política, la teoría del valor, que se va simplificando progresivamente en las sucesivas exposiciones; y en tercer lugar, por la distancia que se presenta entre la autorreflexión de Marx acerca de su propia teoría y el desarrollo efectivo de la misma, que va mucho más allá de su autocomprensión explícita. Por todo ello, estos autores consideran que es necesaria una reconstrucción de la crítica marxiana de la economía política, siendo la piedra angular de dicha reconstrucción los manuscritos preparatorios del $\mathrm{El} \mathrm{Ca}$ pital, a cuya luz hay que interpretar esta obra, completando los vacíos teóricos que se presentan en ella. En este proceso de reconstrucción desempeñarán también un papel importante las categorías de la filosofía hegeliana.

Muchos de los trabajos de Hans-Georg Backhaus están centrados fundamentalmente en analizar las diferencias entre la teoría "marxiana" del valor y la teoría "marxista" del valor, que se remonta a la interpretación de Engels y ha sido la que se ha establecido como dominante en el marxismo ortodoxo. Este autor considera que esta teoría del valor tiene un carácter premonetario, esto es, sostiene que se puede determinar el valor independientemente del dinero. Este carácter premonetario lo tienen también las teorías del valor burguesas, tanto las teorías objetivas del valor de la economía política clásica como las teorías subjetivas del valor de la economía neoclásica, que se constituye a partir de la revolución marginalista y que es la teoría económica que con diversas variantes domina actualmente en la economía académica. Frente a ello, Backhaus considera que la teoría marxiana es una teoría monetaria del valor, lo que significa que su teoría del valor es simultáneamente una teoría del dinero, puesto que la forma de dinero es la forma de valor acabada, es la forma social en el todas las mercancías están referidas de manera omnilateral al dinero como el equivalente general, y esto es necesario para las mercancías puedan referirse unas a otras como valores. Esto es, el dinero no es un mero medio auxiliar para facilitar el intercambio, tal y como considera tanto la economía burguesa como el marxismo tradicional, sino que es una forma económica que se genera de manera necesaria en el intercambio generalizado de mercancías. Ello significa que la teoría marxiana del valor está concebida como crítica de las teorías premonetarias del valor, por lo que caerían también bajo esta crítica las actuales teorías utilitaristas del valor, en la medida en que en este sentido parten de los mismos presupuestos que la economía política clásica. Por su parte, la interpretación de Engels también caería bajo esta crítica de Marx. Evidentemente a Backhaus no le interesa criticar la interpretación de Engels a partir de una comprensión adecuada de la teoría de Marx, algo que en sí mismo no tendría un interés que fuese más allá del puramente historiográfico, sino que su insistencia en las malinterpretaciones de Engels está dirigida a poner de manifiesto en qué medida la interpre- 
tación tradicional de la teoría del valor ha impedido ver realmente el potencial crítico de la teoría de Marx, que es lo que este autor trata de sacar a la luz.

Asimismo, los diversos autores de esta nueva lectura de Marx comienzan a centrarse progresivamente en los problemas internos de la propia teoría marxiana, debidos fundamentalmente a los intentos de Marx de eliminar sus dimensiones metodológicas más complejas e irla haciendo cada vez más popular en las sucesivas versiones publicadas. Uno de los autores que más ha insistido en esta cuestión ha sido Helmut Reichelt, quien señala hacia una progresiva supresión de aspectos metodológicos de la exposición de Marx, lo que da lugar a lo que este autor denomina la "ocultación del método"27. Esto hace que estos autores consideren que la obra fundamental para comprender la estructuración metodológica de la crítica marxiana de la economía política son los manuscritos preparatorios de $\mathrm{El} \mathrm{Capital}^{28}$. Según ellos, en El Capital no tiene lugar el pleno desarrollo de los planteamientos metodológicos contenidos en los manuscritos, sino más bien una simplificación de los mismos, por lo que dichos planteamientos sólo pueden plenamente comprendidos desde los manuscritos y no desde la obra publicada por Marx.

En la línea de la interpretación iniciada por Backhaus y Reichelt desarrollan su interpretación autores como Helmut Brentel, Diethard Behrens, Michael Heinrich o Ingo $\mathrm{Elbe}^{29}$. Estos autores centran gran parte de su investigación en el análisis de las diferencias teóricas fundamentales que existen entre la economía política burguesa y la crítica marxiana de la economía política. Estas diferencias teóricas quedan anuladas desde la interpretación del marxismo tradicional, al eliminar la dimensión crítica de la teoría de Marx y convertir la "crítica de la economía política" en una "economía política marxista", que ya no se distingue de la economía política burguesa en sus fundamentos teóricos, sino simplemente en su orientación política de carácter socialista y en su utilización de la teoría para defender las posiciones de la clase trabajadora.

Como estos autores ponen reiteradamente de manifiesto, la diferencia fundamental entre la concepción marxiana y la de la economía política clásica se sitúa ya al nivel de la teoría del valor, que es comprendida por Marx de una manera esen-

${ }^{27}$ Esta cuestión aparece tratada con la mayor amplitud en su trabajo "Warum hat Marx seine dialektische Methode versteckt", en Beiträge zur Marx-Engels-Forschung. Neue Folge 1996, Hamburg, pp. 73-110.

${ }^{28}$ Fundamentalmente los Grundrisse, y en menor medida también el Urtext zur Kritik der politischen Ökonomie y los Resultate der unmittelbaren Produktionsprozess. Cf. Elementos fundamentales para la crítica de la economía política, cit., y El Capital. Libro I. Capitulo VI (Inédito). Resultados del proceso de producción inmediato, México, Siglo XXI, 2001.

${ }^{29}$ La obra de Heinrich Die Wissenschaft vom Wert y la obra de Elbe Marx im Westen constituyen las aportaciones más importantes de la última generación de teóricos de la "nueva lectura de Marx". 
cialmente distinta a la de la economía burguesa. La teoría del valor-trabajo de la economía política clásica está dirigida a la determinación cuantitativa del intercambio. Parte del trabajo como medida del valor, y a partir de aquí trata de determinar la cantidad de trabajo contenida en las mercancías que se intercambian. La teoría clásica del valor-trabajo, en el más puro espíritu del empirismo anglosajón, funciona así como una teoría de la medida, que pretende establecer la cuantificación directa del trabajo que produce mercancías. Marx, por el contrario, considera el intercambio en primer lugar desde un punto de vista cualitativo, esto es, le interesa la condición de posibilidad misma del intercambio, aquello que hace posible la posible la comparación de objetos cualitativamente distintos, lo cual está ya necesariamente presupuesto a la relación de intercambio. Así pues, Marx no se pregunta en primer lugar por las cantidades que se intercambian, como hace la economía política, sino de qué son cantidades esas cantidades ${ }^{30}$. Y la respuesta que ha dado la economía política, el trabajo, resulta insuficiente para la exigencia teórica de Marx. El trabajo es el metabolismo del hombre con la naturaleza, y en este sentido es algo que han conocido todas las sociedades humanas, pues es la condición de posibilidad de la reproducción social. Este trabajo produce valores de uso, objetos que en su materialidad satisfacen una determinada necesidad. Pero en la sociedad capitalista el valor de uso no es más que el medio de realización del valor de cambio, pues en ella todos los productos son producidos para ser intercambiados. La pregunta a la que está dirigida la teoría del valor de Marx es la pregunta por el modo en que se realiza el metabolismo social es una forma de sociedad en la que todos los individuos están forzados estructuralmente al intercambio. Se trata de una forma de sociedad en la que los individuos realizan su trabajo de manera privada, y sólo a través del intercambio de los productos como mercancías su trabajo vale como trabajo social. Se trata, en definitiva, de una forma de sociedad en la que el intercambio es lo único que produce la conexión social del trabajo. Esta dimensión social del trabajo es algo propio de la sociedad capitalista, y no ha sido conocido por otras sociedades históricas. En esta forma de sociedad, el valor de cambio es la forma social en que se representa el trabajo, y es a esta dimensión cualitativa del intercambio generalizado de mercancías a lo que está dirigida en primer lugar la teoría del valor de Marx.

Considerar que todos los productos del trabajo son tendencialmente mercancías, como hace la economía burguesa, es suponer implícitamente que todos los

\footnotetext{
${ }^{30}$ A este respecto señala Behrens que mientras que la economía política "parte del tiempo de trabajo concreto como spended labour y fijado cuantitativamente, para Marx el tiempo de trabajo no está dado inmediatamente como cuantitativamente determinado" ("Die kritische Gehalt der Marxschen Wertformanalyse", en Behrens, D. (ed.), Gesellschaft und Erkenntnis. Zur materialistischen Erkenntnis und Ökonomiekritik, Freiburg, Ça ira-Verlag, 1993, p. 179).
} 
productos del trabajo están destinados al intercambio, lo que implica introducir subrepticiamente las relaciones sociales capitalistas como relaciones suprahistóricas, que se presentan ya en sus elementos básicos en todas las formas de sociedad. La tendencia natural del hombre sería la intercambiar los bienes que produce ${ }^{31}$, y ello supone proyectar la condición del hombre en la sociedad burguesa, su condición de homo oeconomicus, a todas las sociedades históricas, llevando a cabo así una naturalización de las relaciones sociales burguesas. La crítica de esta naturalización de las relaciones sociales y del fetichismo que necesariamente conlleva será uno de los objetivos fundamentales de la teoría de Marx.

En el análisis marxiano queda claro que la determinación del valor no puede tener lugar en la mercancía tomada aisladamente, por tanto, el valor no puede determinarse a partir del proceso de producción de la mercancía. El valor no es una sustancia que entre en la mercancía a través de su proceso de producción, tal y como considera la economía política y ha sostenido también el marxismo tradicional. Esta concepción del valor es denominada por M. Heinrich "teoría sustancialista del valor”32, y supone obviar la determinación categorial básica de la teoría marxiana del valor. Mantener la coherencia teórica de la argumentación de Marx implica concluir necesariamente que sostiene una teoría no sustancialista del valor. Esto significa que el valor no es una sustancia que le corresponda al producto aislado, sino una sustancia común que sólo se presenta en la relación entre las mercancías en la cual se igualan. Ser valor no es un atributo natural de los productos del trabajo, sino un atributo social. Los productos del trabajo sólo adquieren un valor y se transforman, por tanto, en mercancías, en la relación de intercambio entre ellos ${ }^{33}$. Y sólo en el momento en que las relaciones de intercambio atraviesan toda la sociedad se convierten todos los productos en mercancías, siendo así la mercancía la forma elemental de la riqueza en esta forma de sociedad. Pero esto es sólo así en una formación social en la que las relaciones de cambio se establecen como las relaciones dominantes, teniendo que pasar necesariamente todos los productos del trabajo por el filtro del

${ }^{31}$ Esta concepción se encuentra ya claramente establecida en el origen mismo de la economía política. Adam Smith señala sin ambages que es una "propensión de la naturaleza humana... trocar, permutar y cambiar una cosa por otra" (La riqueza de las naciones, Madrid, Alianza, 1990, p. 44).

${ }^{32}$ Cf. Die Wissenschaft vom Wert, Münster, Westfälisches Dampfboot, 2001, pp. 198 y ss. Sobre esta cuestión, véase también del mismo autor Crítica de la economía politica. Una introducción a "El Capital" de Marx, Madrid, Escolar y Mayo, 2008, pp. 57 y ss.

${ }^{33}$ Esta cuestión la señala Marx explícitamente en distintos pasajes de El Capital, pero el lugar donde la expone con mayor claridad es en el manuscrito que redactó para preparar la segunda edición de El Capital, muchos de cuyos planteamientos no fueron recogidos después en el texto definitivo. Cf. Ergänzungen und Veränderungen zum ersten Band des "Kapitals”, MEGA II.6. (No existe traducción al español de este importante texto). 
mercado, transformándose de este modo en mercancías, y apareciendo junto al contenido material que es el valor de uso, la forma social que es el valor.

Como se ha mostrado anteriormente, la comprensión empirista de Engels y del marxismo ortodoxo lleva en última instancia a una interpretación historicista de la teoría del valor, que considera que Marx está analizando una forma histórica de sociedad anterior a la sociedad capitalista en la que los individuos podían calcular el valor de los productos que intercambiaban a través de un cómputo directo del tiempo de trabajo invertido en ellos. Por lo tanto, la teoría marxiana del valor no sólo supone una crítica a la teoría del valor-trabajo de la economía política clásica, que considera posible el cómputo directo del valor, también caería bajo la crítica de Marx la interpretación dominante del marxismo tradicional, que considera posible igualmente determinar el valor de manera directa en la producción.

La determinación del valor que realiza Marx implica la conclusión de que la sustancia del valor no puede ser en ningún caso una propiedad natural del cuerpo de las mercancías, puesto que es precisamente de estas propiedades de las que se hace abstracción en la relación de intercambio en la cual se igualan las distintas mercancías y en la que se presenta su valor. Tiene que tratarse de una propiedad social, que supone la abstracción de todos los atributos naturales. Si se abstrae de estos atributos, a las mercancías sólo les queda la propiedad de ser productos del trabajo. Pero no se trata del trabajo concreto que produce los cuerpos de las mercancías, pues es precisamente de esta materialidad de la que se hace abstracción en la determinación del valor, por lo que hay que abstraer también de las formas concretas de trabajo, que como tales producen valores de uso, y quedarse con lo que es común a todas ellas, de modo que los distintos trabajos concretos son reducidos en la relación de intercambio en la que se establece el valor a lo que es común a todos ellos, a trabajo humano igual, a trabajo abstractamente humano, en el quedan indiferenciadas todas las formas concretas en que se ha realizado el trabajo. Este trabajo abstracto es precisamente lo que constituye la sustancia del valor ${ }^{34}$. Mientras que el trabajo concreto produce valores de uso cualitativamente distintos que se pueden aprehender materialmente, lo que produce el trabajo abstracto es una objetividad que no se puede reducir a las cualidades materiales del producto. Se trata, en definitiva, de una objetividad social, que no tienen los productos del trabajo tomados por separado, sino que sólo la tienen en común en tanto se refieren los unos a los otros como mercancías. El hecho de que se trata de una objetividad que no se presenta en el objeto tomado aisladamente, hace que Marx hable de una "objetividad espectral (gespenstige Gegenständlichkeit)"35.

\footnotetext{
${ }^{34}$ Mientras que otros aspectos de la teoría del valor aparecen más ampliamente desarrollados en los manuscritos o en la Contribución, la categoría de trabajo abstracto encuentra en El Capital su formulación más completa. Véase El Capital, cit., pp. 51 y ss.

${ }^{35}$ El Capital, cit., p. 47.
} 
En cuanto a este carácter abstracto del valor, es preciso poner de manifiesto que no se trata de una abstracción conceptual realicen los individuos al comparar distintos trabajos concretos, sino que se trata de una abstracción efectivamente realizada en la praxis social de los individuos ${ }^{36}$. En efecto, en la relación de intercambio en la que se igualan las mercancías como valores se abstrae fácticamente de las formas concretas de los distintos trabajos, quedando todos ellos reducidos a lo que les es común, a su carácter abstractamente humano. Para esta forma de abstracción A. Sohn-Rehtel acuñó el concepto de "abstracción real" ${ }^{37}$, del que se han apropiado los teóricos de esta nueva lectura de Marx para su interpretación de la teoría marxiana. Este término como tal no aparece en Marx, pero alude con toda claridad en distintos lugares de su obra a esta dimensión real de la abstracción ${ }^{38}$. Puesto que esta abstracción es resultado de la actuación práctica de los individuos en las relaciones que establecen entre sí en el intercambio, no puede ser reducida a la conciencia que las personas tengan de ella. En efecto, con el surgimiento del modo de producción capitalista, el trabajo abstracto forma parte de la realidad social, con independencia de lo que piensen los indi-

\footnotetext{
${ }^{36}$ Heinrich señala a este respecto que "la «abstracción» de la que aquí se trata no es una abstracción que lleven a cabo conscientemente los individuos que intercambian ... sino que se realiza a través de las acciones de estos individuos" (Die Wissenschaft vom Wert, cit., p. 209).

${ }^{37}$ Sohn-Rehtel afirma en este sentido que el concepto económico de valor "es de naturaleza inmediatamente social, tiene su origen en la esfera espacio-temporal del comercio entre los hombres. No son las personas las que producen esta abstracción, sino sus acciones, sus acciones recíprocas" (Geistige und körperliche Arbeit, Frankfurt a. M., Suhrkamp, 1970, p. 42). En esta obra analiza el autor el modo en que se derivan las formas de pensamiento de los agentes sociales en el sistema capitalista a partir de las relaciones de intercambio, en las cuales se generan las "abstracciones reales" que constituyen la base de la abstracción mental que opera tanto al nivel de la conciencia cotidiana de los individuos como en las teorías económicas y sociales burguesas.

${ }^{38}$ Marx afirma en la Introducción del 57 que las abstracciones a las que se refiere son "verdaderas en la práctica" (Elementos fundamentales para la crítica de la economía política, volumen 1, cit., p. 26) y alude asimismo a la "existencia histórica" de las mismas (ibid., p. 22, p. 24), y en el cuerpo de los Grundrisse señala que dichas abstracciones tienen "una existencia real" (ibid., p. 410). En este mismo sentido afirma en la Contribución que esta "reducción aparece como una abstracción, pero es una abstracción que se lleva a cabo a diario en el proceso de producción social" (Contribución a la crítica de la economía política, cit., p. 18). Asimismo, en el libro II de El Capital afirma que aquéllos "que consideran la autonomización del valor como mera abstracción olvidan que el movimiento del capital industrial es esta abstracción in actu" (El Capital. Crítica de la economía política. Libro II, México, Siglo XXI, 1993, p. 123).

${ }^{39}$ Respecto a ello observa A. Schmidt que "las abstracciones que aparecen en El Capital no son del teórico, sino que se cumplen diariamente en la realidad social. Con el surgimiento de un mundo de mercancías, es decir, con la «forma general de valor» de los productos del trabajo, se convierte el carácter abstractamente general del trabajo humano en su carácter específicamente social" ("Zum Erkenntnisbegriff der Kritik der politischen Ökonomie”, en Schmidt, A.; Euchner, W. (eds.), Kritik der politischen Ökonomie heute. 100 Jahre "Kapital”, cit., p. 33).
} 
viduos integrados en ese sistema ${ }^{39}$. El carácter real de esta abstracción pone de manifiesto que el trabajo abstracto no es una forma de trabajo que se presente en cualquier tipo de sociedad, sino que es la forma social del trabajo efectivamente realizada en la sociedad capitalista. Esta concepción está a la base de la crítica de Marx a la economía política, que atrapada en el fetichismo toma las categorías como si tuvieran validez atemporal.

El fetichismo de las categorías de la economía política es resultado del modo de funcionamiento de la sociedad capitalista, en la que las relaciones sociales se presentan cosificadas, como si fuesen un atributo natural de las cosas, algo inherente a ellas independientemente del contexto social. Pero el hecho de que las cosas tengan propiedades sociales no es algo que ocurra en todas las formas de sociedad, es un fenómeno que sólo tiene lugar en las condiciones de la producción generalizada de mercancías. Esta cosificación de las relaciones sociales es la consecuencia necesaria de la estructura social capitalista, en la que las relaciones de los individuos en sus trabajos no son directas, sino que están mediadas necesariamente por cosas, se constituyen sólo a través del intercambio de los productos del trabajo como mercancías, siendo el mercado la única instancia que establece la conexión social del trabajo. Ello hace que a los individuos les parezca que las cosas siguen sus propias leyes, presentándose ante ellos una instancia de coacción material sobre la que no pueden ejercer ningún control y a la que se encuentran necesariamente sometidos ${ }^{40}$.

Este estado de cosas propio de la sociedad capitalista hace que las personas tengan una conciencia invertida de las relaciones sociales, considerando que son un atributo natural de las cosas. Y esta conciencia fetichista es sistematizada teóricamente por la economía política, en tanto que ciencia que estructura conceptualmente la autocomprensión espontánea de la moderna sociedad burguesa. De este modo, la constitución social e histórica de las relaciones capitalistas permanece oculta tanto para la conciencia espontánea de los agentes sociales como para la economía política, que conforma sus categorías a partir de esas representaciones invertidas. Por ello la teoría social de Marx es simultáneamente crítica de las formas de conciencia fetichista de la sociedad burguesa y crítica de

\footnotetext{
${ }^{40}$ La teoría del fetichismo, que constituye uno de los puntos álgidos de la evolución de la teoría social de Marx, es desarrollada por él en la segunda edición del libro primero de El Capital, mientras que en la primera edición la cuestión del fetichismo aparecía todavía dentro del análisis de la forma de valor, como la cuarta característica de la forma singular de equivalente. Cf. El Capital, cit., pp. 87 y ss. La crítica de la cosificación de las relaciones sociales encuentra su mayor desarrollo en el libro tercero de El Capital, en la denominada "Fórmula trinitaria", donde Marx analiza en profundidad cómo en el sistema capitalista las relaciones sociales aparecen invertidas en propiedades naturales. Cf. El Capital. Libro III, cit., pp. 1037 y ss.
} 
la economía política como ciencia en la que se ha configurado categorialmente la comprensión que dicha sociedad tiene de sí misma ${ }^{41}$.

Lo que se encuentra a la base de la distinción entre la teoría clásica del valor y la teoría marxiana del valor es la diferencia entre la concepción individualista de la economía política y la dimensión social de la teoría Marx. Frente a la economía política, que parte del individuo aislado y considera la sociedad como resultado de las acciones de los individuos (para lo cual proyecta subrepticiamente la estructura social sobre la psicología del individuo), Marx considera que es la estructura social la que establece los parámetros a los que tienen que ajustarse necesariamente las acciones de los individuos. Para Marx no se trata de las decisiones conscientes de los individuos, sino que éstos se encuentran estructuralmente obligados a comportarse así, actúan sin saber realmente lo que hacen, tal y como afirma en la célebre frase de El Capital: "No lo saben, pero lo hacen" La teoría del valor está dirigida de este modo a sacar a la luz los mecanismos de reproducción social en virtud de los cuales el trabajo individual realizado de manera privada puede convertirse en trabajo social, lo cual genera ineludiblemente el fetichismo que se adhiere a los productos del trabajo en tanto que mercancías, y al cual sucumbe tanto la conciencia espontánea de los individuos como la economía política.

Esto lo puede hacer Marx poniendo de manifiesto el carácter doble del trabajo que produce mercancías. Por un lado, el trabajo concreto produce valores de uso, y esta forma de trabajo ha sido realizada en todas las sociedades históricas para poder sobrevivir, es un "necesidad natural eterna". Por otro lado, el trabajo abstracto que constituye la sustancia del valor es la forma históricamente específica de trabajo en una sociedad que se basa en la producción generalizada de mercancías. Al no distinguir entre ambas formas de trabajo, la economía política considera como natural, y como tal propio de todas las formas históricas de sociedad, algo que es exclusivamente social, y que corresponde sólo a una determinada sociedad histórica, la sociedad capitalista. Esta inversión de la forma social del trabajo en natural está a la base de todas las inversiones y mistificaciones de la economía política, y la crítica de esta naturalización fetichista de las relaciones, que lleva a la teoría burguesa tomar las relaciones capitalistas como leyes inmutables correspondientes a todas las sociedades históricas, es el principal objetivo de la teoría del valor de Marx.

${ }^{41}$ En este sentido afirma H. Brentel que la teoría del valor de Marx es "teoría del «fetichismo» de las categorías económicas y al mismo tiempo teoría del carácter fetichista de la conciencia burguesa" (Soziale Form und ökonomisches Objekt. Studien zum Gegenstands- und Methodenverständnis der Kritik der politischen Ökonomie, Opladen, Westdeutscher Verlag, 1989, p. 15).

${ }^{42}$ El Capital, cit., p. 90. 
Todo ello hace patente no sólo la distancia insalvable que separa la teoría marxiana del valor de la teoría clásica del valor, sino también la que la separa de la concepción del marxismo tradicional. La ley del valor es efectivamente para Marx la determinación del valor por el trabajo como su sustancia y su medida inmanente, y esto significa para él la determinación del modo en que tiene lugar la distribución social en una forma de sociedad en la que no existe una organización racional en la distribución del trabajo. La ley del valor es la forma estructuralmente coactiva a través del que se establece, a espaldas de los agentes sociales, la reducción de los distintos trabajos concretos a trabajo abstracto, el cual se constituye así en la sustancia del valor. Esto significa, en definitiva, que los presupuestos de la ley del valor sólo se presentan en un sistema de producción generalizada de mercancías, por lo que se trata de una ley operativa en la sociedad capitalista, no en sociedades precapitalistas como considera Engels y el marxismo tradicional. Con ello se pone claramente de manifiesto que la crítica de Marx de la economía política supone también implícitamente la crítica de lo que tradicionalmente se ha presentado como "marxismo".

\section{BIBLIOGRAFÍA}

Althusser, L.: La revolución teórica de Marx, México, Siglo XXI, 1987

Althusser, L.: Para leer "El Capital”, México, Siglo XXI, 1978

Anderson, P.: Consideraciones sobre el marxismo occidental, México, Siglo XXI, 1991

Backhaus, H.-G.: Dialektik der Wertform. Untersuchungen zur Marxchen Ökonomiekritik, Freiburg, Ça ira-Verlag, 1997

Behrens, D. (ed.): Gesellschaft und Erkenntnis. Zur materialistischen Erkenntnis und Ökonomiekritik, Freiburg, Ça ira-Verlag, 1993

Brentel, H.: Soziale Form und ökonomisches Objekt. Studien zum Gegenstands- und Methodenverständnis der Kritik der politischen Ökonomie, Opladen, Westdeutscher Verlag, 1989

Elbe, I.: Marx im Westen. Die neue Marx-Lektüre in der Bundesrepublik seit 1965, Berlin, Akademie Verlag, 2008

Engels, F.: La subversión de la ciencia por el señor Eugen Dühring (Anti-Dühring), Barcelona, Crítica, 1977

Engels, F.: Dialéctica de la naturaleza, Madrid, Akal, 1978

Engels, F.: Einleitung zur englischen Ausgabe (1892) der "Entwicklung des Sozialismus von der Utopie zur Wissenschaft", MEW 22 (Karl Marx, Friedrich Engels: Werke (MEW), hrsg. vom Institut für Marxismus-Leninismus beim Zk der SED, Berlin, 1956 ff.)

Euchner, W.; Schmidt, A. (eds.): Kritik der politischen Ökonomie heute. 100 Jahre "Kapital”, Frankfurt a. M., Europäische Verlagsanstalt, 1968 
La evolución teórica del marxismo: del materialismo histórico a la crítica de la conciencia fetichista

Heinrich, M.: Die Wissenschaft vom Wert. Die Marxsche Kritik der politischen Ökonomie zwischen wissenschaftlicher Revolution und klassischer Tradition, Münster, Westfälisches Dampfboot, 2001

Heinrich, M.: Crítica de la economía política. Una introducción a "El Capital” de Marx, Madrid, Escolar y Mayo, 2008

Korsch, K.: Marxismo y filosofia, Barcelona, Ariel, 1978

Lenin, V. I.: Materialismo y empiriocriticismo, Madrid, Ayuso, 1974

Lukács, G.: Historia y conciencia de clase, Barcelona, Grijalbo, 1976

Mandel, E.: Tratado de economía marxista, México, Era, 1976

Marx, K.: Manuscritos: economía y filosofia, Madrid, Alianza, 1997

Marx, K.: Elementos fundamentales para la crítica de la economía política (Grundrisse) 1857-1858 (volumen 1), México, Siglo XXI, 2001

Marx, K.: Elementos fundamentales para la crítica de la economía política (grudrisse) $1857-$ 1858 (volumen 3), México, Siglo XXI, 2002

Marx, K.: Contribución a la crítica de la economía política, México, Siglo XXI, 1998

Marx, K.: El Capital. Crítica de la economía política. Libro I, México, Siglo XXI, 1995

Marx, K.: El Capital. Crítica de la economía política. Libro II, México, Siglo XXI, 1993

Marx, K.: El Capital. Crítica de la economía política. Libro III, México, Siglo XXI, 1996

Marx, K.: El Capital. Libro I. Capitulo VI (Inédito). Resultados del proceso de producción inmediato, México, Siglo XXI, 2001

Marx, K.: Ergänzungen und Veränderungen zum ersten Band des "Kapitals”, MEGA II.6 (Karl Marx, Friedrich Engels: Gesamtausgabe (MEGA), hrsg. vom Institut für Marxismus-Leninismus beim Zk der KPdSU und vom Institut für Marxismus-Leninismus beim Zk der SED; seit 1990: hrsg. von der Internationalen Marx-Engels-Stiftung (Amsterdam), Berlin, 1975 ff.)

Marx, K.; Engels, F.: La ideología alemana, Barcelona, Grijalbo, 1974

Postone, M.: Tiempo, trabajo y dominación social: una reinterpretación de la teoría crítica de Marx, Madrid, Marcial Pons, 2006

Reichelt, H.: Zur logischen Struktur des Kapitalbegriffs bei Karl Marx, Freiburg, Ça-Ira Verlag, 1970

Rosdolsky, R.: Génesis y estructura de "El Capital” de Marx. Estudios sobre los "Grundrisse”, México, Siglo XXI, 1985

Rubin, I.: Ensayos sobre la teoría marxista del valor, Buenos Aires, Pasado y Presente, 1974

Schmidt, A. (ed.): Beiträge zur marxistischen Erkenntnistheorie, 1969

Schmidt, A.: El concepto de naturaleza en Marx, México, Siglo XXI, 1983

Smith, A.: La riqueza de las naciones, Madrid, Alianza, 1990

Sohn-Rehtel, A.: Geistige und körperliche Arbeit, Frankfurt a. M., Suhrkamp, 1970

Zeleny, J.: La estructura lógica de "El Capital” de Marx, Barcelona, Grijalbo, 1974 